pregnancy, but it may, possibly, inhibit to some extent activity of the uterine muscle. Its clinical value is greatest in the first few weeks of pregnancy in virtue of its effect on the decidual tissue; there is little evidence of its value later on in the human being.

It is impossible to discuss within the limits of this article the full therapeutic use of ovarian hormones or the treatment of dysfunction in general, though mention has been made of one or two conditions in which they may be employed successfully. Many cases of dysfunction respond to general medical and dietetic measures, and some respond to thyroid administration. The pituitary and ovarian hormones should be employed only after most careful consideration and, if necessary, full detailed investigation. Commercial preparations of synthetic oestrogens are now so inexpensive that these have perhaps been employed without full appreciation of their probable value. Haphazard employment is to be condemned, for harm, rather than good, may result. The first line of attack on menstrual disorders is a general examination of the patient; such examination may demonstrate the need for measures other than hormone preparations.

\title{
THE DIFFICULTIES AND DANGERS OF THE PRIMIPAROUS WOMAN
}

\author{
By MORRIS DATNOW, F.R.C.O.G. \\ (Hon. Asst. Surgeon, Liverpool Maternity Hospital, and Women's Hospital, Liverpool) \\ Introduction
}

Most obstetrical problems are common to all women, multiparae and primigravidae alike, there are, however, features which can be regarded as special to potential mothers carrying their first child, and it is with these clinical conditions that this paper is essentially concerned.

The care of the primigravida is yet the obstetrician's most urgent and anxious problem, for her safe delivery prevents many undesirable sequelae, leading to sterility, disability, injuries, invalidism, and a fear of bearing more children. This psychological state is difficult to rectify and naturally harmful to the national economy. In practice many women are encountered who only have or had one child, and state that they had such a bad time in the confinement that they really could not face another, although they would very much have liked a family.

There has been considerable progress in antenatal care and diagnosis, especially with refer-. ence to X-ray examinations, biological tests, also advances in obstetrical technique, improved anaesthesia, operative technique, greater institutional facilities with more prolonged teaching and training of both midwives and students, and post-graduate courses. New drugs and also improved old ones. All these factors must play their part in lessening morbidity and mortality. Tocergometry, hysterography and the counting of labour pains are more recent investigations which have been interrupted by prevailing conditions, and the future will possibly demonstrate their value as the results observed so far already indicate.

Notwithstanding the amount of the clinical research that has been carried out there are as yet many unknowns to cause anxiety during pregnancy and labour for the primigravida. Hospital facilities for the lying-in woman are far from sufficient, and there still is a tendency to foster domiciliary midwifery in districts which only have antenatal clinics supervised by general practitioners without adequate qualification for this very specialised branch of medical practice. In some localities primigravidity is not looked upon as sufficient indication for institutional delivery. This is greatly to be deprecated, especially with the imperfect home help that is available at present. 


\section{Statistics}

The following table (I) figures the difficulties and complications encountered at the Liver- 3 pool Maternity Hospital summarised from the Annual Reports for the ten-year period I932- $\varrho$ r94I, both years inclusive. The patients who were delivered in the district are taken into. account.

TABLE I

\begin{tabular}{|c|c|c|c|c|c|c|c|c|}
\hline & & & & & Primigravida & $\begin{array}{l}\text { Per cent of } \\
\text { total deliveries }\end{array}$ & Multigravida & $\begin{array}{l}\text { Per cent of } \\
\text { total deliveries }\end{array}$ \\
\hline \multirow{2}{*}{\multicolumn{2}{|c|}{$\begin{array}{l}\text { Forceps . . } \\
\text { Albumen with convulsions }\end{array}$}} & $\ldots$ & .. & $\ldots$ & $\mathrm{I}, 554$ & $3 \cdot 97$ & 269 & $0 \cdot 68$ \\
\hline & & .. & . & . & 699 & $\mathbf{I} \cdot 77$ & 441 & $I \cdot 12$ \\
\hline \multicolumn{2}{|c|}{ Caesarean section } & . & .. & . & 658 & $1 \cdot 66$ & 965 & $2 \cdot 47$ \\
\hline Induction of labour & .. & .. & . & .. & $57 \mathrm{I}$ & $\mathrm{I} \cdot 45$ & 885 & $2 \cdot 24$ \\
\hline Breech presentation & . & & . . & . & 450 & $I \cdot I_{4}$ & 389 & $0 \cdot 98$ \\
\hline \multicolumn{4}{|c|}{ Antepartum haemorrhage (accidental) } & . & 197 & $0 \cdot 50$ & 269 & 0.68 \\
\hline \multicolumn{2}{|c|}{ Postpartum haemorrhage } & $\ldots$ & . & . & 175 & $0 \cdot 44$ & 234 & $0 \cdot 59$ \\
\hline Multiple pregnancy & $\cdots$ & .. & . & . & 160 & $0 \cdot 40$ & 180 & $0 \cdot 45$ \\
\hline \multicolumn{2}{|c|}{ Heart disease $\ldots$} & $\therefore$ & . & $\therefore$ & 148 & $0 \cdot 37$ & I 77 & $0 \cdot 44$ \\
\hline \multicolumn{5}{|c|}{ Antepartum haemorrhage (placenta praevia) } & I I 7 & $0 \cdot 29$ & 289 & $0 \cdot 73$ \\
\hline Eclampsia $\quad \ldots$ & .. & $\ldots$ & $\ldots$ & . & 66 & $0 \cdot 17$ & 28 & $0 \cdot 07$ \\
\hline Version .. & .. & .. & . & . & 54 & $0 \cdot 12$ & 148 & $0 \cdot 37$ \\
\hline Retained placenta & . & . & . & .. & 53 & $0 \cdot 12$ & 50 & $0 \cdot 12$ \\
\hline Face $\ldots$ & 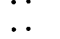 & 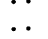 & $\ldots$ & $\ldots$ & 45 & O. I I & 56 & 0.12 \\
\hline Prolapse of cord & .. & .. & . & .. & 37 & 0.09 & 78 & $0 \cdot 19$ \\
\hline Shoulder $\quad \ldots$ & . & $\ldots$ & .. & . & I 7 & $0 \cdot 04$ & 47 & $0 \cdot 11$ \\
\hline Brow $\quad \ldots$ & .. & .. & . & . & 12 & 0.03 & 14 & 0.03 \\
\hline
\end{tabular}

In an evaluation of the above figures due regard must be had to the fact that a largeg number of primigravidae are treated in hospitals, as are also, of course, difficult cases generallg, but this factor is not really likely to lead to gross exaggeration of the natural incidence becauge $\overrightarrow{0}$ abnormal multiparae will be concentrated in institutions as well. Another compensatim factor as far as these figures are concerned is brought about by the fact that the old multipapie is now increasingly recognised as being a potential case of difficulty for whom there is con-O siderable danger in labour as was first stressed by Professor Leyland Robinson in 1930, and referred to in the June number of this Journal. There is now a greater tendency generally to hospitalise these patients rather than allow them to be confined at home.

Looking through the above list it is obvious that most of the complications are common to the multipara and primigravida alike, although the individual problems are naturally more involved for the primigravida.

The total number of patients that attended the hospital for the years under review was 39,363. Excepting breech presentations, most of the other abnormal presentations are more frequent in multigravida with an especially high preponderance for transverse lie and prolapse of the cord, facts which are universally accepted.

The high figures for primigravida who had forceps applied, the occurrence of albumenuria and eclampsia, will require further scrutiny, and these conditions will be commented upon later.

\section{Analysis of Obstetric Difficulties}

The simplest way to analyse and summarise the obstetric difficulties, dangers and problems $\frac{D}{0}$ of the primigravida is to consider them as groups with either a constitutional or a local effect, just as would be the case for the multigravida, although the general statement must always be $N$ reiterated that difficulties are more frequent in primigravida, and also perhaps have more $N$ serious consequences for these women.

Constitutional factors that have an influence on or are disturbed by pregnancy.

The following classification summarises and systematically sets out the constitutional factors that have an influence on or are disturbed by pregnancy. 
TABLE II

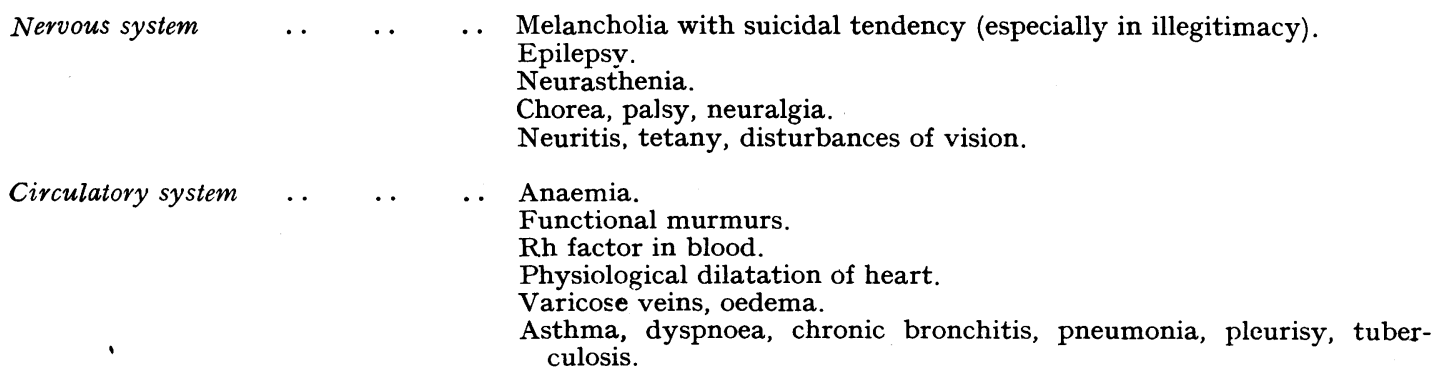

Hormonopoetic system _. $\quad$. . Deficiency of lutein.

Disorders of the pituitary.

Hyperthyroidism.

Altered supra renal function.

Disorders of the pancreas.

Excretory system $\quad \ldots \quad \ldots \quad \ldots \quad$ Alimentary tract disorders.

Urinary tract disorders-pyelitis, nephrolithism.

Toxaemias _. $\quad \ldots \quad \ldots \quad \ldots \quad$. $\quad$ Pre-eclampsia, albumenuria, eclampsia.

Accidental antepartum haemorrhage, abortions, bacterial infections and zymotic diseases.

Other toxaemias.

Metabolic disturbance. . . . . . Calcium deficiency.

Vitamin deficiency-other nutritional disturbances.

\section{Local factors that have an influence on or are disturbed by pregnancy.}

\section{TABLE III}

$\begin{array}{lll}\text { Anatomical derangements } \ldots & \ldots & \begin{array}{l}\text { Underdevelopments. } \\ \text { Malformations, misplacements, trauma, pruritis and other dermatoses, } \\ \text { torsion of the uterus, volvulus. }\end{array} \\ \text { Physiological factors and disorders } \ldots & \begin{array}{l}\text { Placenta praevia. } \\ \text { Hydramnios. }\end{array} \\ & \begin{array}{l}\text { Hydatidiform mole. } \\ \text { Foetal abnormalities and deformities. } \\ \text { Ectopic gestation, abnormal presentations, multiple pregnancy, } \\ \text { uterine inertia, contraction ring and other uterine dysfunctions. }\end{array}\end{array}$

Tumours $\quad \ldots \quad \ldots \quad \ldots \quad \ldots \quad$ Innocent neoplasms of the uterus and ovaries.

Malignant neoplasms.

Tumours of the placenta and membranes.

Tumours of the pelvic bones.

Infections $\quad \ldots \quad \ldots \quad \ldots \quad \ldots \quad$.

Appendicitis.

Pyelitis.

Peritonitis.

It is not proposed to consider all the conditions adumbrated above. Nothing new nor that is not generally known about these factors has appeared, and it will be sufficient to dwell only on those subjects that will be helpful to primigravida in the present state of knowledge. They are:-

I. The toxaemias of pregnancy, eclampsia and albumenuria.

2. The estimation of mechanical difficulties with prevention of trauma.

3. Evaluation of the strength of the uterine tone and contractions.

4. Conditions developing after confinement with especial reference to the control of puerperal infection.

5. Obstetrical operative procedures. 


\section{Control of toxaemias}

Extended antenatal supervision of pregnant women undoubtedly has had a marked influence in reducing the incidence of toxaemia. The figures for toxaemia are still high in the table of abnormalities of pregnancy as is seen in Table I, which has been compiled from the ten-year period during which antenatal clinics had already become well established and routine, illustrating the fact that the examinations made and treatment given fail wholly to eliminate the toxaemias, and that it is still a problem for the primigravida.

There are no new developments in the diagnosis of toxaemia. Their incidence is anticipated by routine blood-pressure readings, weighings of the patient, examination of the urines, and the education of the patient to recognise early signs and symptoms, such as oedema, headaches, disturbances of vision and digestion. This instruction of the patient has had a tendency to make a few apprehensive, but on the whole the patient is very co-operative.

Now there is a tendency to group eclamptics into types: viz. renal, hepatic, neural and mixtures of these.

Blood pressure, albumenuria, oedema with increase of weight remain the essential diagnostic features. Liver and kidney function tests are of no great negative value, whereas positive results are usually present in advanced and serious cases with gross kidney and liver damage. During the war period a slightly higher percentage of cases of eclampsia have occurred very early in pregnancy before the twentieth week. The greater incidence of illegitimacy with the extra mental and physical strain due to present exigencies no doubt have some bearing on this.

\section{Treatment of Toxaemias.}

The principles of treatment mainly followed by authors are still those based on metabolism and elimination, and to a less extent the control of abnormal stimuli to the central nervous system.

The work of Stroganoff, though frequently quoted, has not become popular in this country, ${ }^{*}$ nevertheless it has an application in special cases, for example the intra- and post-partum eclampsias.

Water balance, control of the fluid intake to correspond to the quantity of water passed, restriction of chlorides, as this is raised in the blood of eclamptics, oestrin induction and rupture of the membranes for bringing on labour are the main advances that are concerned with treatment. Methods of reducing blood pressure by blood letting, veratrone, and either intravenous or intrathecal injections, or both of hypertonic solutions should be mentioned, although the extended use of magnesium in this connection is rather questioned by Muduliar and others as they have shown an increase in the blood magnesium of eclamptics, especially marked in the fatal cases.

The value and importance of institutional treatment is now appreciated by all.

\section{Mechanical difficulties}

a. Breech.-The estimation of mechanical difficulties and the recognition of abnormal presentations and their correction during pregnancy has now become a routine matter, assisted by the extended use of X-rays in pregnancy. Clinically it must be accepted that head presentations give the best results for mother and child in primigravidae, although the work of Potter in America and the observations of Burns in Liverpool on the management of breech confinements have given a helpful lead. Version should be undertaken 4-6 weeks before full term with the patient fully anaesthetised if necessary. The danger of separation of the placenta is negligible, and premature onset of labour does not matter because the patient will have an easier time and the child is well viable. Spinal, sacral and local anaesthesia, and also episiotomy are useful in breech deliveries. If version fails in elderly primiparae Caesarean section becomes a necessity, especially when the legs of the foetus are extended, as these patients tend to become post-mature as well, with an added risk to rupture of the tentorium cerebelli in the less mouldable foetal head.

* In this connection attention should be drawn to the work of Mudaliar, A., Mayor, R. S. M., and Minon, M. K. K., J.O.B.E., 47, 1940, 291, who state that they have halved their previous mortality figures (16.7 per cent to 8.75 per cent) by adopting Stroganoff's method, together with artificial rupture of the membranes; 79 per cent of their patients ceased to have fits after the liquor was permitted to escape. 
$b$. Disproportion.-In doubtful cases the ability of the head to pass through a particular pelvic brim and then to negotiate its way out cannot be estimated clinically with certainty before labour, so it might be of some value to examine this question. The gross method of inserting a finger (Pinard) between the head and the symphysis is sufficient to prove that a head will pass with certainty. In doubtful cases the Moller-Munro-Kerr method is followed, and, finally, trial labour is the only sure way to discover how much moulding is going to take place.* The methods available therefore are essentially clinical, and this skill is only acquired by practice.

c. Pelvimetry.-Advances are taking place in X-ray pelvimetry and cephalometry which in time may be universally available, and possibly become routine for the primigravida. In this connection the work of Caldwell and Molloy should be mentioned as the most recent reclassification of the types and shapes of human pelves morphologically with an attempt to standardise and extend the knowledge concerning the difficulties due to the passages in labour, thus helping in the prognosis of the mechanism of labour especially in primigravidae. These authorities essentially studied the shape and form of the pelvis, and evolved four parent types. By X-ray observations in over a thousand cases during labour, the manner in which forceps are to be applied and the levels at which rotation should be attempted were postulated by Molloy and his co-workers.

In their hospital it has been possible to diminish the difficult forceps cases without increasing the number of Caesarean sections undertaken. A knowledge of the mechanics and Caldwell's suggestions will lead to better handling of forceps with improved foetal mortality and maternal morbidity and mortality in primigravidae.

An accurate measurement of the pelvic inlet and outlet can now be made by the radiologists provided with suitable apparatus, the position is still, however, that the clinician should carefully observe the progress of labour and apply the appropriate treatment. Most, if not all, primigravidae in which there is the slightest doubt about the pelvis should be admitted to an institution, and all maternity units should have a modern X-ray installation with the co-operation of an experienced radiologist.

The growing practice of doctors lulling themselves into a false position of security by having their patients X-rayed at some time in the pregnancy and relying on the radiologist's report to dogmatise about the confinement being normal is much to be deprecated, and one would venture to state that good clinical obstetrical acumen is as desirable as an X-ray picture, which at best is only a record of what the state of affairs is during a particular flash.

\section{Evaluation of the strength of uterine contractions}

Modern views on uterine action in labour tend to presume that oestrin produces hyperaemia with an actual sensitising action to the oxytocic pituitrin. Theoretically the assumption is that in the primigravida the muscle of the uterus should be in a prime stage of activity. In practice, however, it is found to be liable to the same dysfunctions that are encountered in those who have borne many children, viz., (i) primary uterine inertia, (ii) secondary uterine inertia, (iii) contraction ring, and (iv) physiological inertia. The fourth type of inertia is most frequently encountered when there are abnormal presentations or there is disproportion.

\section{Inertia Dystocia.}

Clinically, in labour, the effectiveness of uterine contractions are estimated by the degree of taking up and dilatation of the cervix with the descent of the presenting part. The frequency and severity of pain are not always a gauge, although the length of contraction is an important factor. It is possible by experience to judge the strength and effectiveness of the uterus by palpation during contractions. In this connection it must be pointed out that in every individual labour the length and force of pains vary, so that it is advisable to feel several successive contractions before attempting to prognose.

\section{Methods of estimating the strength of the uterine muscle.}

Many attempts have been made to estimate mechanically and more scientifically the

* In this connection it must be pointed out that sufficient time should be allowed for the head to descend, or rather it is best to wait until the patient has strong regular contractions of the uterus because in doubtful cases the head frequently remains high at the onset of labour, whilst the pains are still infrequent and of very short duration. 
strength of uterine contractions during pregnancy, the most recent and apparently successful is Collier's method of tocergometry. During labour many more investigations have been carried out to estimate the strength of the uterus which vary from the intra-uterine introduction of a metroreunter into the uterine cavity and recording the intra-uterine pressure-internal hysterography - to the application of a machine to the abdominal wall and recording alterations brought about by the contracting uterus-external hysterography. In both instances the work was chiefly undertaken to test out the action and effect of drugs and anaesthetics.

Embrey describes an apparatus by means of which he had been able to obtain representative tracings of the uninfluenced uterus, and points out that uterine inertia or inefficiency may be demonstrated by two types: the first shows contractions of feeble intensity and duration recurring at too frequent intervals causing little discomfort. In the second the contractions are colicky and painful, of quite irregular intensity, duration and frequency, and are ineffective in bringing about dilatation of the maternal passages and expulsion of the child.

Sandor Lorand demonstrated his Tokograph at the International Congress in 1938, and was able to show a number of tracings which point to the value of this instrument in estimating the strength of uterine contractions and the reaction of the uterus to drugs which are exhibited to the mother.

Clinically attention is drawn to weakness of the muscle by a flabby uterus during pregnancy which fails to respond to ordinary stimulation such as palpation and pulling on the nipple, a low blood pressure and diminished blood calcium. Blair Bell advocated the use of calcium by mouth during the pregnancy, and pituitrin injections during the latter weeks of pregnancy, and more recently the intravenous injection of calcium gluconate has been suggested.

Biological blood analyses have not become popular because they require a fair quantity of blood for each examination, and take a long time to complete. Simple tests which can be applied by the clinician, or are easily available to him, will facilitate matters in the future. A history of dysfunction as evidenced by dysmenorrhoea, scanty menstruation, and difficulty in conceiving have some influence in assisting the clinician to form an opinion on uterine function in labour when taken together with other clinical features.

\section{Dystocia dystrophia.}

Recently Bryan Williams has again drawn attention to the dystocia dystrophia syndrome⿳亠丷厂 first recognised by de Lee. He gives an incidence of $\mathrm{I}-80$ for primiparae. Attention is draw to the fact that age is not an important factor in this condition. Slightly contracted pelvis, masculine type, menstrual irregularity and illegitimacy, narrow introitus and vagina, apprehension of difficulty on the part of the primigravida should be added, postmaturity, failure to go into labour with any form of induction near term should place the obstetrician on guard. Restlessness with a desire to push in the first stage, a cry for relief soon after the onset of labour, slow cervical dilatation with an inability to relax the muscles generally. Transverse engagement of the head with slow descent and very little caput succedaneum. Forceps are invariably required. The babies are larger than average, the patient's stature is short, obese with male characteristics for distribution of the hair on the body.

\section{Contraction ring dystocia.}

Contraction ring dystocia is a condition which is recognised when fully established by a local spasm of the uterus visible per abdomen, usually opposite to the neck of the child, and prevents its expulsion. Traction with forceps fails to allow of delivery because when traction is made the uterus contracts and grips the child, extraction can only be made between pains with deep anaesthesia or relaxation obtained by means of amyl nitrite, and in some cases Caesarean section is the only method of delivery.

Attention was first drawn to the prophylactic treatment of this condition by Professor Miles Phillips, who states that its onset is preceded by a longer or shorter period of painful and ineffective activity of the uterus described by the term colicky action of the uterus. This colicky action can be recognised by the fact that the painfulness of the uterine contraction persists after the palpable hardening has passed away. Treatment of this colicky action with repeated doses of morphia until it has ceased will prevent the onset of a contraction ring and allow of normal delivery.

The fact that there is a high incidence of one child sterility in the dystrophia cases is not 
necessarily due to the general factors, because in practice doctors sometimes advise these patients not to have any more children, an unreasonable thing to do, and to be deprecated. The patients who have had a rather difficult time during labour naturally have a fear of a repeat experience, and time should be spent in reassuring them and so adjust their psychology. Finally, infection may lead to closure of the tubes and sterility. Those patients who do conceive again have been delivered quite normally, subsequently pointing, perhaps, to the fact that this condition of dystrophia is not entirely due to pelvic structure, and would be better included amongst the physiological inertias.

\section{Conditions developing after confinement}

Post-natal care and examination is the way to prevent many women who have borne their first child from having to live a somewhat restricted and uncomfortable life with the constant fear of future conception, as all their symptoms appear to date from the birth of the child. It is therefore advisable not to relax supervision after the confinement, but to continue meticulously to supervise the puerperium. Frequent emptying of the bladder, regulation of the bowels, constant changes of posture especially sitting up and prone positions. In this connection the book of Miss Randall's on exercises Training for Childbirth from the Mother's Point of View will repay reading. A primigravida should not be allowed out of bed too soon after her confinement, as she is inclined to overdo, and it is better to over-restrain her rather than allow her to commence household duties too soon. She should be impressed with the fact that it takes four to six weeks to recover sufficiently to be able to breast feed a child as well as to look after the husband and house. A vaginal examination should always be made four to six weeks after delivery, and any misplacements corrected and kept right by means of instruments and posture. At this time also the primigravida will have a number of questions to ask concerning her difficulties and fears. Such an examination gives her an opportunity of receiving the necessary advice, thus relieving her anxiety.

In this way it is possible to prevent and alleviate such common symptoms as backache, frequency of micturition and dysuria, constipation, piles, vaginal discharges, blood-stained and otherwise, lack of proper sleep, loss of appetite with indigestion, faintness and anaemia with constant depression. Douching should not be undertaken in the puerperium.

When there has been a complicated pregnancy or puerperium, a longer period of convalescence should be enforced. Superlactation with the hope of preventing pregnancy has no foundation, and retards normal recovery.

When symptoms persist after a carefully supervised puerperium, these should be investigated and treated. In this connection the easy access of a convalescent home is valuable. The war has also demonstrated that it is quite safe to move women fairly soon after delivery by ambulance without any risk to them, especially after normal confinements.

Good post-natal care will help to relieve the gynaecological and orthopaedic clinics as much as do ante-natal clinics and good supervision during labour. The restriction of visitors during the lying-in period is an advantage.

The paragraph would be incomplete without just brief mention of puerperal sepsis. The outlook in this condition has been greatly altered by the advent of blood antiseptics and chemotherapy. There is no need to enter into discussions on this matter because so much has been written, but it would be timely to just draw attention to the fact that resort to chemotherapy should not be made before full investigation and enquiries into the causes of temperature in every case have been undertaken first. The use of sulphanilamide as a diagnostic agent is dangerous. For the recognition of the methods of infection, types of organism, and methods of treatment, the writing of Lancefield, Hare, Colebrook, Paine, Cruikshank, and many others can be consulted.

\section{Treatment}

Recent advances in treatment are chiefly concerned with-

I. Induction of labour by rupture of the membranes, by intra-amniotic injections of various substances and by the exhibition of oestrin parentally or by injection.

2. Lower segment Caesarean section.

3. Improvement of oxytocic drugs.

4. Anaesthesia, and

5. Blood transfusion. 
Rupture of the membranes has very little general application for primigravida. Stimulation of uterine contractions and onset of labour at first brought about accidentally during investigations with intra-amniotic injections cannot have any vogue by design, especially for women pregnant with their first child. Stilboestrol and similar preparations are helpful to stimulate the uterus when sluggish and in cases at term or over, especially where a raised blood pressure precludes the use of pituitrin or thymophysin. Acetylcholine has not come up to what is expected of it theoretically.

For a number of years the routine use of quinine, castor oil and pituitrin induction in various combinations has led to the conclusions that medicinal induction is safe. In primigravida, if employed during the last week of pregnancy, it will cause the head to descend and so exclude disproportion when this is in doubt. It helps to diminish the number of many of the difficulties described above, especially with regard to improving uterine tone. The more frequent passage of meconium by the babies is no cause for undue alarm, and the use of forceps is not grossly more frequent than with primigravida in general. In this connection it is appropriate to draw attention to the value of a pair of light straight forceps with a long shanks and fairly large handle for use when the head has not quite rotated but has passed the mid straight. If forceps are to be applied in the primigravida, use should be made of these at the earliest possible time in the interests of the child, following preliminary ironing out of the vagina and distension of the introitus.

The facility with which whole blood, serum and dried serum can now be obtained has contributed greatly to the improvement of the anaemias and bleedings of pregnancy. The discovery of the $\mathrm{Rh}$ factor, now being more fully investigated, is elucidating the causes of some of the previously less explainable stillbirths, neonatal diseases, and fatalities. The blood of all patients with these complications should be sent for test. All cases where a second blood transfusion is necessary at an interval after the first should be shown to be Rh positive, or else $\mathrm{Rh}$ negative blood will have to be obtained. There is as yet no method of preventing intrauterine death of the foetus when the maternal blood is Rh negative and the father's Rh positive.

The work of Marshall on the lower segment Caesarean section has brought about standardisation of the technique and extension of the indications for operative delivery. On the other hand, the lower segment operation has made more possible that very valuable aid to obstetricians, namely, trial labour, and in favourable surroundings this is the most rational way to determine whether a certain head will pass through the brim, especially in the primgravida.

\section{BOOK REVIEWS}

\section{ACUTE INJURIES OF THE HEAD}

By G. F. Rowbotham. With a foreword by Norman M. Dorr. Pp. 288, with 124 illustrations. Edinburgh: E. \& S. Livingstone. 1942. 25 s. net.

The object of the book, as stated in the preface, is that it is primarily for those who are responsible for the treatment of acute cerebral trauma and those who have not received a special training in neuro-surgery.

While the theories advanced are not to be wholeheartedly acclaimed, the practice advised is both acceptable and commendable. Nevertheless to recommend the generalised adoption of those methods well established in the hands of the author would be to court disaster in the hands of those unaccustomed to neuro-surgical methods.

Many equivocal points arise. The mechanisms of fracture-production propounded are interesting but their constant application is doubtful. That "suction" plays any considerable part in injuries of the brain distant to the site of impact is no convincing, when the intracranial pressure $i$ usually sub-atmospheric, and accordingly maintains normal apposition of the cranial contents. The infrequency with which aerial bombardment leads to penetrating injuries is not the common impression, nor can one accept the statement that cerebral injury may arise out of a tangential blow by a bullet in the absence of scalp injury. Does escape of blood cells by "diapedesis" account for contusion?

Difficulties in diagnosis of closed cerebral injuries are well known to those seeing large numbers of head injuries, and these are not simplified by statements such as "giddiness, mental confusion, or stupor is pathognomonic of extra-dural compression," and "a fixed dilated pupil indicates raised intracranial pressure of such a degree that it will lead to a fatal outcome if not relieved." Such comments could lead to many negative explorations.

The course of investigation recommended and the treatment prescribed are clearly and ably described. Once more it may be said that what is well-founded in the author's practice is not altogether advisable for universal employment. Amongst the list of indications for Encephalography is the persistence 